\title{
P03-255
}

\section{QUALITY OF LIFE AMONG THE NAVIES AT SIX MONTHS AFTER THE TSUNAMI IN PHANG-NGA NAVAL BASE, PHANG- NGA PROVINCE, THAILAND}

\section{R. Kongsakon}

Dep. of Psychiatry, Faculty of Medicine Ramathibodi Hospital, Bangkok, Thailand

Objective: This study aimed to identify the health-related quality of life (HRQOL) among the navies or their spouses at 6 months after the tsunami disaster, to compare HRQOL with the 2006 Thailand normative data for Short Form - 36 (Bangkok) ${ }^{4}$.

Method: A structured questionnaire modified from the 36-item Medical Outcome Study Short-Form Health Survey (MOS SF - 36), including demographic data, characteristics and amount of loss, social support, health information, and type of Tsunami exposure were recorded.

Result: A total of 434 subjects participated in this study. Male was $77 \%$. Married was $67 \%$. Mean age was $33.56 \pm 9.26$ years old. The QOL scores among members of the Navy or their spouses were lower than the 2006 Thailand normative data (Bangkok) in almost all subscales except physical functioning, bodily pain, and social functioning. The major consequence of the Tsunami was mental health problems. A majority of the survivors who seriously experienced mental problems were female, low education, decreased income, loss of one's family members/loved ones, or property. Bodily pain was the only indicator subscale of QOL which was significant statistically associated with Tsunami exposure.

Conclusion: The QOL measurement provided essential information that helped healthcare providers to identify survivors' needs and outcomes. In addition, the changes in QOL over time after Tsunami disaster might be an effective guideline for health resources allocation. To improve their quality of life, these people required appropriate social support as well as physical and mental health care. 